\title{
Intense Outreach: Experiences Shifting University Students’ Identities
}

\author{
Brean Prefontaine, ${ }^{1}$ Claudia Fracchiolla, ${ }^{2}$ Manuel Vasquez, ${ }^{3}$ and Kathleen Hinko ${ }^{1}$ \\ ${ }^{1}$ Department of Physics and Astronomy, Michigan State University, East Lansing, MI, USA \\ ${ }^{2}$ Marie Curie Fellow, University College Dublin, Dublin, Ireland \\ ${ }^{3}$ JILA, University of Colorado Boulder, Boulder, CO, USA
}

\begin{abstract}
In addition to research and classes, physics students may choose to participate in informal physics teaching experiences; however, these programs are understudied as part of the physics student experience. We investigate university educators' (UEs) negotiation of physics identity after they participate in an informal program for K-12 students as part of the Science Theatre student group at Michigan State University. We hypothesize that that the UEs' science identity is reshaped by the interactions and experiences they have in these programs, especially an intensive week-long trip to the Upper Peninsula. Pre- and post- interviews were collected with Science Theatre participants who went on the spring break trip. In analyzing this data, we demonstrate the efficacy of using a Community of Practice framework to understand UEs' experiences as they negotiate their memberships in the outreach and scientific communities.
\end{abstract}

\section{INTRODUCTION}

Physics education research has been concerned in the past decade with understanding the identities of physics students. The findings of these identity studies have been used to suggest ways to leverage formal classroom learning to increase belonging and potentially create a more inclusive physics culture [1]. Focusing on formal environments makes sense from an instructor perspective; however, what has been largely overlooked are the informal experiences where undergraduate and graduate students freely choose to interact with physics outside of their coursework. Informal learning is differentiated from formal learning by students' degree of agency and voluntary participation, making them potentially very powerful experiences for identity [2]. Opportunities for informal learning at the college level include student-led physics groups, which are commonly funded through physics departments, that often allow students to interact with physics content and practices through public outreach. In fact the American Physical Society's officially sanctioned student organizations, Society of Physics Students and Sigma Pi Sigma, list "outreach services to the campus and local communities" in their mission statement [3].

In our previous work, we have looked at the motivation and the preparation that university educators (UEs) — who are university students participating in some form of teaching or facilitation in informal environments - in a K-12 afterschool physics program called PISEC, run through the University of Colorado Boulder $[4,5]$. These studies showed that the experiences students have while participating in these type of activities can have a positive impact on their attitudes towards outreach. One important aspect was that many students devoted a significant amount of time and effort to the PISEC outreach group, participating for multiple semesters and even years and taking on more leadership roles.

Based on these findings, we postulate that certain physics groups that engage in informal teaching can function as communities of practice, especially if the informal activities are designed to be particularly intense, immersive, or meaningful. In this paper, we apply the Community of Practice frame- work to examine the experiences and identity of members of an undergraduate physics/science demonstration group at Michigan State University (MSU). We present a case study of a student who demonstrates changing membership and increased competence after participating in a unique week-long outreach trip with the group over spring break. Through application of this theoretical model on an informal physics program, we also demonstrate the importance of informal teaching and learning physics communities for students.

\section{COMMUNITY OF PRACTICE FRAMEWORK}

The Community of Practice (CoP) framework was developed in 1991 by Lave and Wenger to identify "groups of people who share a concern or a passion for something they do and learn how to do it better as they interact regularly" [6]. In the physics education research community, it has recently been used to look at the Learning Assistant experience [7] and students in advanced lab courses [8]. In order to be considered a CoP, a group must have three attributes: some shared goal or expertise (domain), ways to help each other achieve the goals (community), and have a set of norms, common repertoire, and shared information to achieve the domain (practice) [6].

An individual's membership in a CoP is affected by their competence. In this case, competence is defined by the community and what it values through engagement and participation. There are dimensions of competence for a CoP: $a c$ countability to the enterprise, mutuality of engagement, and negotiability of the repertoire. These dimensions of competence become dimensions of identity in the sense that they help determine the degree of membership of the individuals within a CoP. That is, a competent member - a central member of the community - is able to achieve those three dimensions. Apart from the dimensions of competence, Wegner also identified different categories that link CoP to identity such as negotiated experiences, learning trajectories, and nexus of multimembership. These categories determine what experiences within the $\mathrm{CoP}$ help one become a more com- 
petent, central member of that community. In this work, we focus on the competence of individuals within the CoP; we do this because the consideration of individuals' membership levels allows us to probe the nature of the CoP itself, as well as individuals' identities.

The accountability to the enterprise construct is related to what members value and do based on their membership and considers how members understand the goals/objectives of the community, how they take responsibility and embrace those goals, how they contribute to the achievement of those goals, and their constant negotiation and redefinition of the goals. An example would be when members of a group talk about enjoying group activities.

The mutuality of engagement construct is about how members interact with other members in the community and is the basis for identity through participation. Included here are how members engage with other members to establish relationships within the community. For example, this would be seen when a more senior member of the community helps a newer member get up to speed.

The negotiability of the repertoire construct is related to how members may think about their ability to use skills, language, and resources of the practice to engage with the community. For instance, this can be seen when a group member talks about a specific demo that may be done during an outreach event. Related to identity, this construct translates into a personal set of events, references, memories, and experiences that create relations of negotiability with respect to the repertoire of the practice.

\section{SCIENCE THEATRE CONTEXT}

We investigate membership ideas in the context of a student group called Science Theatre at MSU. This student organization is funded by the physics department and consists of undergraduate and graduate students aiming to "get children and adults alike excited about the wonders of science" [9]. In order to achieve this, the group has developed physics and other science demonstrations that are used in both stage shows (generally for an audience of local K-12 students) and hands-on environments (i.e. at the MSU science festival). Most events are one-time and between 1-2 hours in length. Students in the group can choose to participate in as many of the one-time events as fits their schedule. There are $40 \mathrm{stu}-$ dent volunteers from a variety of STEM majors in this group some of whom have officer roles, a staff coordinator, and a physics faculty advisor. They have a large multi-room working space in the basement of the MSU planetarium to store equipment, create and test new demos, and to socialize.

One unique aspect of Science Theatre is the opportunity for members to participate in an intensive outreach experience during a spring break road trip throughout the Upper Peninsula of Michigan. For this week long trip, volunteer MSU Science Theatre members drive demonstration equipment to northern rural Michigan and put on stage shows at K-
12 schools who have limited contact with universities. They spend Monday through Friday traveling from school to school in 3-4 person teams to do presentations during both morning and afternoons. In the evenings the teams reconvene to eat dinner and debrief on the day. During spring 2018, nine students (eight undergraduates and one graduate) and the staff coordinator did 55 presentations during this one week trip.

We postulate that Science Theatre is a CoP that has the potential to support the physics identities of its members. From our knowledge of the group (via conversations with students, observations of both group meetings and outreach events, and extended communication with the staff and faculty advisors), we can identify the three essential aspects of a CoP (domain, community, and practice) within Science Theatre. The students share the domain of getting the general public excited about science, a community of both undergraduate and graduate students who are able to hold one of the twelve officer positions, and a practice that consists of a wide range of demonstrations and scripted shows [9].

We further hypothesize that participation in the spring break trip can be a highly impactful activity for Science Theatre members. Due to the nature of the trip, all of the participants are together the entirety of the trip (driving, doing shows, and rooming together at hotels). This immersion allowing for many social interactions among group members is another reason why we believe the $\mathrm{CoP}$ framework is an appropriate choice.

\section{METHODS}

Working from the externally supported claim that Science Theatre is a CoP, we seek to determine the competence of its participants. To accomplish this objective, we designed a qualitative study where we interviewed participants about their experience in both Science Theatre and the spring break trip. During spring 2018, 10 matched interviews (5 pre-trip and 5 post-trip) were collected from undergraduate students participating in the Science Theatre spring break trip. All of the students were interviewed during the two weeks leading up to spring break and again during the two weeks following the trip. The semi-structured interviews followed a pre-trip protocol that focused on competence related to their involvement with Science Theatre, future career plans, and expectations of the trip. The post-trip protocol focused on feelings about the trip, specific demos performed, and the impact of going on the trip.

To test the efficacy of the CoP framework through the competence constructs, we have chosen to look closely at an interview set from a unique case of a freshman psychology student (referred to as Matt). This particular student was chosen for several reasons, including his relatively short Science Theatre history (due to being a freshman student) and a more peripheral membership prior to the trip than other interviewees (due to having no stage show experiences before the trip and not being in an officer position within the club). While this stu- 
TABLE I. Emergent themes and example quotes for competence constructs. Post-trip interview quotes have been denoted with an asterisk.

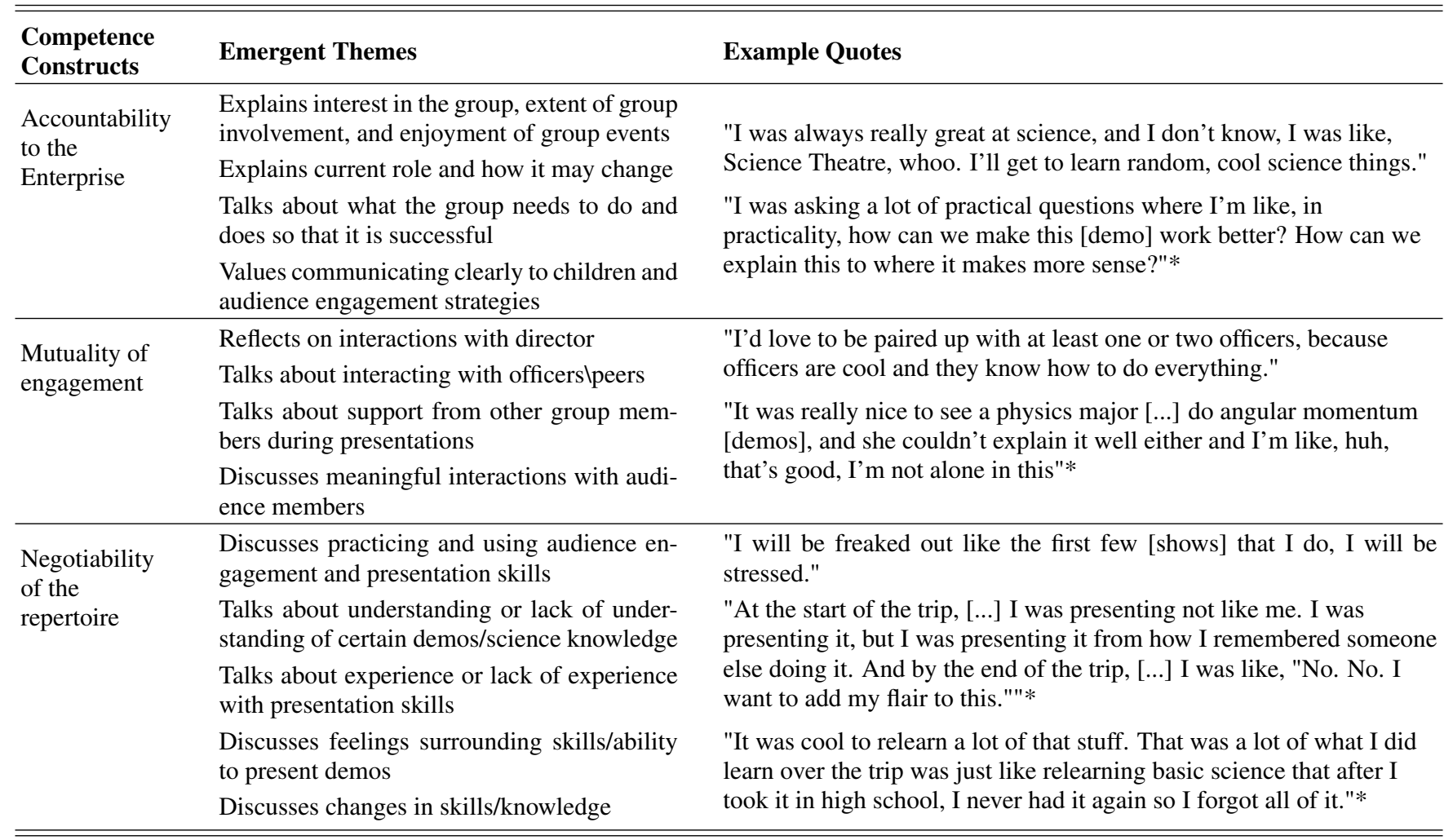

dent does not have a physics background, he does express his enthusiasm for the goals of the group by actively choosing to participate in physics activities outside of his major while also showing tension about his lack of physics knowledge. Throughout the interviews, he shared extensively and openly about his feelings and experiences related to being a part of the group and participating in the physics activities. Thus, we found Matt's interviews to be especially rich for exploring how competence was shown in his growing membership within Science Theatre.

Two researchers analyzed the interviews by coding with the three competence constructs. During the process, we classified our findings as being high, medium, or low confidence in terms of how well it fit into the constructs. This process helped us refine our operationalized codes and is part of our validation process. We also included a fourth category called "other things of interest" that was a place for us to identify portions of the interviews that may have been interesting but did not necessarily fit with the other three constructs. All codes from the pre and post interview were discussed and all discrepancies between the two researchers were resolved.

\section{FINDINGS}

From our analysis, we find all three of the competence constructs were present in both interviews. Table 1 summa- rizes each construct with corresponding emergent themes and example quotes. The emergent themes are evidence of the contextualization of the constructs within this data set. The accountability to the enterprise construct successfully highlighted Matt's involvement and role within the group, his thoughts about the group's success, and his own development of thoughts surrounding science communication. The mutuality of engagement construct allowed for Matt's reflections about interactions with other members of the CoP to be brought into focus. Finally, the negotiability of the repertoire construct brought to light Matt's acquired skills and knowledge as well as his feelings surrounding this growth.

Moreover, we find that the competence constructs allowed us to characterize Matt's membership within Science Theatre, recognize changes in his membership, and identify mechanisms for these changes. A comparison of the example quotes shown for accountability to the enterprise in Table 1 make it evident that Matt became more accountable to the Science Theatre enterprise throughout the spring break trip by taking the time to think deeply about the presentation and the audience's interactions with the demonstrations. This increased accountability was also evident when Matt shared his intention of running for the geoscience officer position. However, when asked if he had any intention of running for the position before the spring break trip, he mentioned that he had not, but that during the trip he had the following interaction with John, who is a Science Theatre student director: 
"John just came up to me [and said] "Matt, you should do geoscience because you'd be great at it." And I was just like, wait. [...] The fact that he thinks I would make a really good geoscience director is kind of amazing. Because he knows that I'm not super involved in Science Theatre and he knows I'm not a geoscience major but he still thinks that I'd be doing a really good job at it. So that opinion was what made me think, dang, apparently I've got something going on that other people don't."

This interaction with John seemed to provide Matt the confidence he needed to run for an officer position. Thus we see that through this interaction and his mutuality of engagement, Matt is becoming more accountable to the enterprise (by wanting to devote more time as an officer) and his competence is increasing (as he is actively attempting to move toward a more central membership role within the group).

Throughout the interviews, Matt explains the skills he deems necessary to be successful during Science Theatre events. He talks about presentation and audience engagement skills, his own science knowledge, and knowledge of various different demos. For example, the quote presented in Table 1 from the post-trip interview related to mutuality of engagement shows Matt relating to one of his peers struggling with the physics content knowledge even though she is studying physics. This interaction leads to his reflection of how much science knowledge he has gained within his repertoire. Another instance of Matt reflecting on his growing repertoire was present during the post-trip interview when he was asked about the most challenging portion of the trip:

"[Before the trip] I know what all of these demos are, but I don't know how to present any of them. I know how to present two and we have like 25 demos or something ridiculous. And then through the week, I picked up more and more demos in my repertoire until at the end of the week it was like every single demo that does fire, I know how to do it."

This reflection on the challenging task of learning the various demos that he needed to present during the trip shows Matt's acknowledgment of the growth of his presentation skills. This quote was coded as negotiability of the repertoire because it shows Matt shifting from just having knowledge of Science Theatre's set of demonstrations to then being able to confi- dently present the demos that have been added to his skill set. These instances of Matt reflecting on his increased repertoire are evident of a mechanism of his gaining competence.

From this evidence of the CoP framework and contextualization of the competence constructs, we plan to code the remaining interviews in the data set. In this work, we have used to competence constructs to take a close look at the Science Theatre community. Recognizing that the students in our data set are likely members of many communities, we plan to use community codes that will distinguish between the informal experience, field of study, scientific research, and other communities. We have also noted that the competence codes allow for identification of changes in membership within a certain community, and thus plan to create an additional layer of subcodes within the competence codes in order to identify quotes that demonstrate positive experiences, or 'inbound' movement (moving toward becoming a more central member of the group), and negative experience, or 'outbound' movement (moving toward becoming a more peripheral member). By flagging the quotes that demonstrate inbound and outbound movement as well as identifying the communities that are being discussed, we hope to take a closer look at the possible intersections, membership, and identity across multiple communities. We also recognize that the nature of the Science Theatre spring break trip is unique and so this work is part of a larger study that looks at programs with many different formats in order to identity what type of experiences have larger impacts on participants.

\section{CONCLUSIONS}

In this work, we have shown that applying the Communities of Practice framework to an informal physics program provides insightful results related to the competence of the community members. We have shown that the competence codes within the framework are successful at highlighting subtleties within the experiences of the students that may point to changes of their identity. With additional analysis, we will be able to use the competence codes to identify the unique features of Science Theatre that allow for a CoP. From there, we will be able to discuss ways that physics outreach can be organized in order to foster development of identity and competence within the group. Thus, future work may lead to understanding best practices for other informal physics groups. This work was supported by NSF \#1423496.
[1] Hazari, Z., et al. The Physics Teacher Vol. 5 Issue 2. Jan 2017.

[2] Nat'1 Research Council. Learning Science in Informal Environments: People, Places, and Pursuits. 2009.

[3] https://www.spsnational.org/about. Retrieved July 5, 2018.

[4] Fracchiolla, C., et al. PERC Proc., Sacramento, CA. July 2016.

[5] Bennett, M., et al. PERC Proc., Cincinnati, OH. July 2017.
[6] Lave, J. and E. Wenger. Situated Learning: Legitimate Peripheral Participation. 1991.

[7] Close, E., et al. PERC Proc., Portland, OR. July 2013.

[8] Irving, P. W. \& Sayre, E. C. Phys. Rev. ST Phys. Educ. Res., Vol. 10 Issue 1. 2014. American Physical Society.

[9] https://web.pa.msu.edu/sci_theatre/. Retrieved April 12, 2018. 